\title{
Apoptosis in human unfertilized oocytes after intracytoplasmic sperm injection
}

\author{
Liana Bosco, Ph.D., ${ }^{\mathrm{a}, \mathrm{c}}$ Giovanni Ruvolo, Sc.D., ${ }^{\mathrm{b}}$ Giovanni Morici, ${ }^{\mathrm{c}}$ Maurizio Manno, Sc.D., ${ }^{\mathrm{b}}$ \\ Ettore Cittadini, M.D., ${ }^{\mathrm{a}}$ and Maria C. Roccheri, Sc.D. ${ }^{\mathrm{c}}$
}

\begin{abstract}
${ }^{a}$ Dipartimento Materno-Infantile, University of Palermo; ${ }^{b}$ Centro di Biologia della Riproduzione, Palermo; and ${ }^{\mathrm{c}}$ Dipartimento di Biologia Cellulare e dello Sviluppo, University of Palermo, Palermo, Italy
\end{abstract}

\begin{abstract}
Objective: To investigate the presence of programmed cell death in unfertilized oocytes after intracytoplasmic sperm injection (ICSI), assuming that previous apoptotic events could be correlated with the fertilization failure. Design: Comparison of the rate of DNA fragmentation in human oocytes at different stages of maturation soon after pick-up (control) and in unfertilized oocytes after ICSI treatment.

Setting: In vitro fertilization (IVF) laboratory with extensive ICSI experience.

Patient(s): Sixty-three patients undergoing assisted fertilization by ICSI.

Intervention(s): Terminal deoxynucleotidyl transferase-mediated digoxigenin-dUTP nick-end labeling (TUNEL) assay and anticaspase-3 cleaved immunoassay to detect apoptosis in control and ICSI-treated oocytes.

Main Outcome Measure(s): Differences in the percentage of oocytes demonstrating DNA fragmentation between control oocytes and unfertilized ICSI treated oocytes at different stages of maturation.

Result(s): The DNA fragmentation, by TUNEL assay, appeared in all the immature control oocytes, but only $37 \%$ of mature oocytes showed DNA fragmentation. This DNA fragmentation was observed in $88.8 \%$ of the oocytes unfertilized after ICSI; furthermore, DNA fragmentation appeared as well in the sperm injected into the cytoplasm.

Conclusion(s): The study has shown DNA fragmentation in human oocytes unfertilized after ICSI. The evidence is confirmed as well in control oocytes, free from in vitro culture or manipulation stress. Caspase-3 immunoassay suggests the presence of apoptosis. The high percentage of oocytes demonstrating DNA fragmentation in the unfertilized oocytes could be correlated with fertilization failure. (Fertil Steril ${ }^{\circledR}$ 2005;84:1417-23. (02005 by American Society for Reproductive Medicine.)
\end{abstract}

Key Words: Apoptosis, fertilization failure, intracytoplasmic sperm injection, ICSI, human oocytes, virgin oocytes

Apoptosis, or programmed cell death, is a process that eliminates superfluous or genetically compromised cells. This process involves a series of metabolic pathways that lead to substantial modifications in the cell oriented toward programmed death, so it has an important role in the normal functions of all tissues. Apoptosis has been recognized as a determining factor in limiting abnormal cellular proliferation and maintaining the homeostasis strategy that controls the number of cells during development and differentiation.

The apoptotic processes eliminate cells with irreversible DNA damage that is harmful for the organism. This process is used by organisms during development and is highly conserved (1). Several studies have demonstrated that the development of healthy embryos is closely related to and coordinated by the process of oocyte maturation before ovulation $(2,3)$. It has been demonstrated that the oocyte maturation, both in vitro and in vivo, depends on the cytoplasm

Received December 29, 2004; revised and accepted May 3, 2005. Supported by MIUR (60\%), and Dottorato di Ricerca in Biotecnologie Applicate alla Medicina della Riproduzione e alla Medicina Perinatale. Reprint requests: Maria C. Roccheri, Sc.D., Dipartimento di Biologia Cellulare e dello Sviluppo "A. Monroy," Università di Palermo, Viale delle Scienze, ed. 16, 90128 Palermo, Italy (FAX: +39-091-657-7430; E-mail:mariroc@unipa.it). and on nuclear maturation as well as on their synchronism. A very important role in this process is played by the cumulus cells through the gap junctions, which allow a physical connection between the cumulus cells and the oocyte. This hypothesis is supported by studies (4) carried out on the granulosa cells of women who have become pregnant after in vitro fertilization and embryo transfer (IVF-ET).

It has been demonstrated that the percentage of apoptotic cells in women who achieved pregnancy was considerably fewer compared with women who did not become pregnant. Moreover, DNA fragmentation in oocytes associated with apoptotic evidence might be one of the reasons for poor oocyte quality and lower fertility in aged mice (5). Of greater concern would be discerning whether early apoptotic events are present in human oocytes treated with advanced techniques of assisted reproduction, in particular with intracytoplasmic sperm injection (ICSI), in which a single sperm is injected into an oocyte. The ICSI technique is now accepted as a specific treatment for infertile patients with a serious seminal deficiency (severe oligoasthenoteratozoospermia and azoospermia) $(6,7)$ and has provided good results in terms of fertilization and pregnancy rate (8). Although ICSI is a very invasive technique that bypasses all the physiologic barriers that the oocyte normally provides for selecting the 
sperm that will penetrate its cytoplasm, it does not allow fertilization in all the mature oocytes treated as only $70 \%$ of the microinjected oocytes are fertilized (8-10). A hypothesis that could justify this fertilization deficit should consider that one or both the gametes may carry anomalies. The most probable defects could be due to an incorrect chromatin condensation or the presence of damage in the sperm DNA, as some investigators have already demonstrated in pathologic studies on semen samples (11-13).

Other investigators $(14,15)$ have demonstrated chromosomal anomalies in the oocytes; an increase in such anomalies seems to be related to the patient's age and exposure to cigarette smoke (16). The oocytes, like spermatozoa, may contain fragmented or damaged DNA independent of the number of chromosomes, as has been demonstrated in the rat (5). The aim of our study was to demonstrate the presence of damaged DNA in unfertilized oocytes after ICSI treatment, considering that apoptotic events in these cells could occur before treatment and thus lead to fertilization failure.

\section{MATERIALS AND METHODS}

This study was approved by the scientific committee of the Dipartimento Materno-Infantile of the University of Palermo, Italy.

\section{ICSI Procedure}

Oocytes were obtained from 63 patients participating in an assisted fertilization program. Starting from day 21 after the last menstrual period (long protocol), $200 \mu \mathrm{g}$ of acetate buserelin (Suprefact; Hoechst, Hounslow, United Kingdom) were administered per day, and stimulation was provided during the treatment menstrual cycle using recombinant follicle stimulating hormone (recombinant FSH; Serono, Geneva, Switzerland). Human chorionic gonadotrophin (hCG; Serono), 10,000 IU, was administered when the follicle maximum diameter had reached $17 \mathrm{~mm}$. The oocytes were collected 36 hours later.

The oocytes, isolated from the follicular fluid, were incubated in culture medium (IVF; Vitrolife, Kungsbacka, Sweden) at $37^{\circ} \mathrm{C}$ in $5 \% \mathrm{CO}_{2}$. Approximately 2 hours after the aspiration, the oocytes were transferred to a medium containing hyaluronidase $(80 \mathrm{IU} / \mathrm{mL}$; Medicult, Jyllinge, Denmark) to remove the cells of the cumulus-corona complex. The naked oocytes were incubated in a culture medium at $37^{\circ} \mathrm{C}$ in $5 \% \mathrm{CO}_{2}$ until the ICSI procedure. Only oocytes that had extruded the first polar body (MII stage) were microinjected. The oocytes were transferred in $10-\mu \mathrm{L}$ drops (one for each drop) of culture media (Gamete 20; Vitrolife), approximately $5 \mu \mathrm{L}$ of polyvinylpyrrolidone (PVP; Medicult), in which the spermatozoa, previously isolated by centrifugation at $300 \times g$ for 7 minutes and then washed in Gamete 20, were suspended. All of the drops were set on the cover of a Falcon Petri dish and were covered with mineral oil (Medicult).
The microinjection was carried out using an inverted microscope (Olympus, Tokyo, Japan) at magnification $\times 400$ on which two micromanipulators (Instruments, Tokyo, Japan) were mounted that allowed the micromovements of two needles on a three-dimensional plane. After ICSI, the oocytes were transferred in fresh culture medium (G1; Vitrolife) and incubated at $37^{\circ} \mathrm{C}$ in $5 \% \mathrm{CO}_{2}$. After 18 hours, the two pronuclei were observed; those that showed a second polar body extrusion, which is evidence of fertilization, were recovered. The oocytes that did not show these signs were isolated for the study. After 48 hours, the presence of cleaved embryos (with two or four blastomeres) was observed. The unfertilized oocytes, usually discarded, were used for our study.

\section{TUNEL Assay in Human Oocytes}

Terminal deoxynucleotidyl transferase-mediated digoxigenindUTP nick-end labeling (TUNEL) assay was performed on 165 oocytes: 81 unfertilized after 48 hours from the microinjection (ICSI), and 84 "virgin" oocytes. The latter, originally destined to be discarded soon after oocyte retrieval, were donated after informed consent by patients who had decided against oocyte freezing. Each oocyte was transferred in drops of paraformaldehyde $3.7 \%$ under paraffin oil in Petri caps for 60 minutes at room temperature. They then were washed three times in PBS 1X $(137 \mathrm{mM} \mathrm{NaCl}, 2.68 \mathrm{mM}$ $\mathrm{KCl}, 1.47 \mathrm{mM} \mathrm{KH}_{2} \mathrm{PO}_{4}, 8.1 \mathrm{mM} \mathrm{NaH} \mathrm{PO}_{4}$ ) and permeated for 10 minutes in ice in Triton X100 $0.1 \%$ and sodium-citrate $0.1 \%$ in PBS $1 \mathrm{X}$. After being washed three times for 5 minutes with distilled water, treated oocytes were incubated for 60 minutes at $37^{\circ} \mathrm{C}$ in a humidified chamber in $50 \mu \mathrm{L}$ of a solution containing reagents (Amersham Biosciences, Amersham, United Kingdom): TdT buffer, Cy3-dCTP (0.3 nmol), "cold"-dCTP (0.033 nmol), TdT enzyme (10 U), and distilled water.

The negative controls were performed incubating some oocytes with the same TdT solution without TdT enzyme, and the positive controls were performed using oocytes pretreated for 5 minutes with $100 \mu \mathrm{L}$ of DNAse-buffer (40 $\mathrm{mM}$ Tris- $\mathrm{HCl}$ at $\mathrm{pH} 7.9,10 \mathrm{mM} \mathrm{NaCl}, 6 \mathrm{mM} \mathrm{MgCl}_{2}, 10 \mathrm{mM}$ $\mathrm{CaCl}_{2}$ ) and then for 10 minutes in a DNAse-buffer solution containing $10 \mathrm{unit} / \mathrm{mL}$ of DNAsi I. The reaction was blocked with $\mathrm{NaCl} /$ sodium-citrate (300 mM-30 mM NaCitrate) for 30 minutes, then the oocytes were washed three times (for 5 minutes each) using PBS. All oocytes were stained with propidium iodide $(1 \mu \mathrm{g} / \mathrm{mL})$ for 10 minutes.

All treated oocytes were transferred on polylysine slides, five per slide, in $10 \mu \mathrm{L}$ of DABCO solution $(233 \mathrm{mg}$ of DABCO in $0.8 \mathrm{~mL}$ of $\mathrm{H}_{2} \mathrm{O}$ and $0.2 \mathrm{~mL}$ of Tris- $\mathrm{HCl}$, with 9.0 $\mathrm{mL}$ of glycerol added) to maintain the fluorescence, and then were observed under a confocal microscope Olympus FV300, with a He-Ne $(543 \mathrm{~nm})$ laser.

The TdT assay showed DNA fragments generated as an effect of the cut from the endogenous endonuclease, acti- 
vated in the last stage of the apoptotic process. The exogenous enzyme TdT recognizes the free extremity $3^{\prime} \mathrm{OH}$ of the fragmented DNA and adds a modified fluorescent deoxynucleotide, Cy3TM-dCTP, to identify the single apoptotic cells (17-19). As a consequence of the reaction, chromatin appears red when positive for apoptosis.

\section{Anticaspase-3 Cleaved Immunoassay}

Another 10 MII oocytes were used for the caspase-3 cleaved polyclonal antibody immunoassay. Each oocyte was transferred in drops of paraformaldehyde $3.7 \%$ under paraffin oil in Petri caps for 60 minutes at room temperature. After being washed three times in PBS $1 \mathrm{X}(137 \mathrm{mM} \mathrm{NaCl}, 2.68 \mathrm{mM}$ $\mathrm{KCl}, 1.47 \mathrm{mM} \mathrm{KH} \mathrm{PO}_{4}, 8.1 \mathrm{mM} \mathrm{NaH} \mathrm{PO}_{4}$ ), they were permeated for 10 minutes in ice in Triton X100 $0.1 \%$ and sodium-citrate $0.1 \%$ in PBS $1 \mathrm{X}$.

After three rinses with PBS 1X, the oocytes were allowed to incubate overnight with caspase-3 cleaved polyclonal antibody (Cell Signaling Technology, Beverly, MA) (1:50 dilution) in PBS containing 3\% bovine serum albumin (BSA). In the negative control oocyte, the primary antibody was omitted. As secondary antibody, anti-rabbit Ig fluoresceinlinked (Amersham) (1:60 dilution) was added to the samples for 1 hour after three rinses with PBS. The secondary antibody surplus was eliminated by washing 3 times with PBS. All oocytes were stained with propidium iodide $(1 \mu \mathrm{g} / \mathrm{mL})$ for 10 minutes. Then they were transferred on polylysine slides, five per slide, in $10 \mu \mathrm{L}$ DABCO solution to maintain fluorescence. Finally, they were mounted on coverslips and observed under a confocal microscope Olympus FV300, with a He-Ne (543 nm) laser.

\section{Statistics}

The results in the study and control group were compared using the $\chi^{2}$ test. All reported values are given as $95 \%$ confidence interval. $P<.05$ was considered statistically significant.

\section{RESULTS}

To demonstrate the presence of programmed cell death in unfertilized oocytes after ICSI treatment and to verify that apoptotic events can occur previously in these cells, we carried out in situ a TdT assay and anticaspase- 3 cleaved immunoassay.

The oocytes, five per slide, were treated with TdT assay as described previously. The observation under the confocal microscope (Olympus FV300) showed that, of the 18 immature virgin oocytes, 12 were germinal vesicles $(\mathrm{GV})$ and 6 were in metaphase I (MI), both GV (Fig. 1A); the majority of MI oocytes (83.3\%) showed evident signs of DNA fragmentation. Of the remaining 66 virgin MII oocytes, 24 (37\%) showed signs of chromatin fragmentation, and 42 (63\%) did not show signs of fluorescence with the TdT assay because of the integrity of chromatin. These data appear to be very important because they could justify the $30 \%$ of oocytes that are not usually fertilized by the ICSI technique, as demonstrated in the literature.

Observations under the confocal microscope of the $81 \mathrm{MII}$ oocytes not fertilized after ICSI treatment showed that 72 oocytes $(88.8 \%)$ with fluorescent chromatin, a sign of fragmentation (see Fig. 1B). Moreover, fragmentation was present in the nemaspermic chromatin of six oocytes (see Fig. 1C); in three of them, it was present in both the oocyte and the sperm chromatin (see Fig. 1D), which shows that DNA fragmentation also is present in injected sperm. Thus, of the 81 oocytes studied (after ICSI treatment), only 9 $(11.2 \%)$ did not show fragmentation in the oocyte chromatin. All the data concerning these experiments are described in Table 1.

In addition, we performed some control studies. To verify the presence and the integrity of the chromosomes, we stained the oocytes that did not show fluorescence after TUNEL procedure with propidium iodide. Figure 1E shows a MI oocyte after propidium iodide staining; the same oocyte was negative to TUNEL assay. As a further control of TdT assay, we performed positive and negative control studies using unfertilized oocytes after ICSI, as described previously. In particular, the positive controls were represented by oocytes pretreated briefly with DNAse I to induce partial DNA fragmentation, as confirmed by the successive observation under the confocal microscope that the oocyte and sperm chromatin appeared fluorescent (see Fig. 1F). The negative controls were obtained by not adding to the reaction mix the TdT enzyme, so they did not show fluorescence (not shown).

Another 10 oocytes were used to carry out a further immunoassay to support the idea that the DNA fragmentation obtained from the TdT assay was an apoptotic event. Immunologic tests were performed in situ with the anticaspase-3 cleaved antibody, an effector caspase. Ten oocytes, unfertilized after ICSI, were observed in metaphase II. Figure 2 shows an MII oocyte after ICSI in apoptosis, as revealed by in situ immunoreaction with caspase- 3 cleaved antibody (see Fig. 2A) and counterstaining with propidium iodide (see Fig. $2 \mathrm{~B}$ ). The merged image is shown in Figure 2C. The negative control, performed on an another oocyte incubated without caspase-3 antibody showed no cytoplasmic fluorescence; the same oocyte was counterstained with propidium iodide, and the merged image is shown in Figure 2D.

All the immunoreacted oocytes (100\%) showed the presence of cleaved caspase-3, a sign of irreversible apoptosis.

\section{DISCUSSION}

The role and control of programmed cell death in human oocytes are still unknown. In the ovary, massive cell death occurs during fetal life and postbirth life as an integral part of normal ovarian development. Ovarian cellular death is an 


\section{FIGURE 1}

Apoptosis evaluation using TUNEL assay in human oocytes. The images show equatorial sections observed through confocal laser microscopy. (A) Germinal vesicle positive for TUNEL. (B) MII oocyte with evident signs of apoptosis. (C) Apoptotic sperm chromatin in a MII nonfluorescent oocyte. (D) MII oocyte after ICSI treatment, with apoptotic male and female chromatin. (E) MI oocyte after propidium iodide staining. (F) Positive control of assay. Scale bar $=15 \mu \mathrm{m}$.
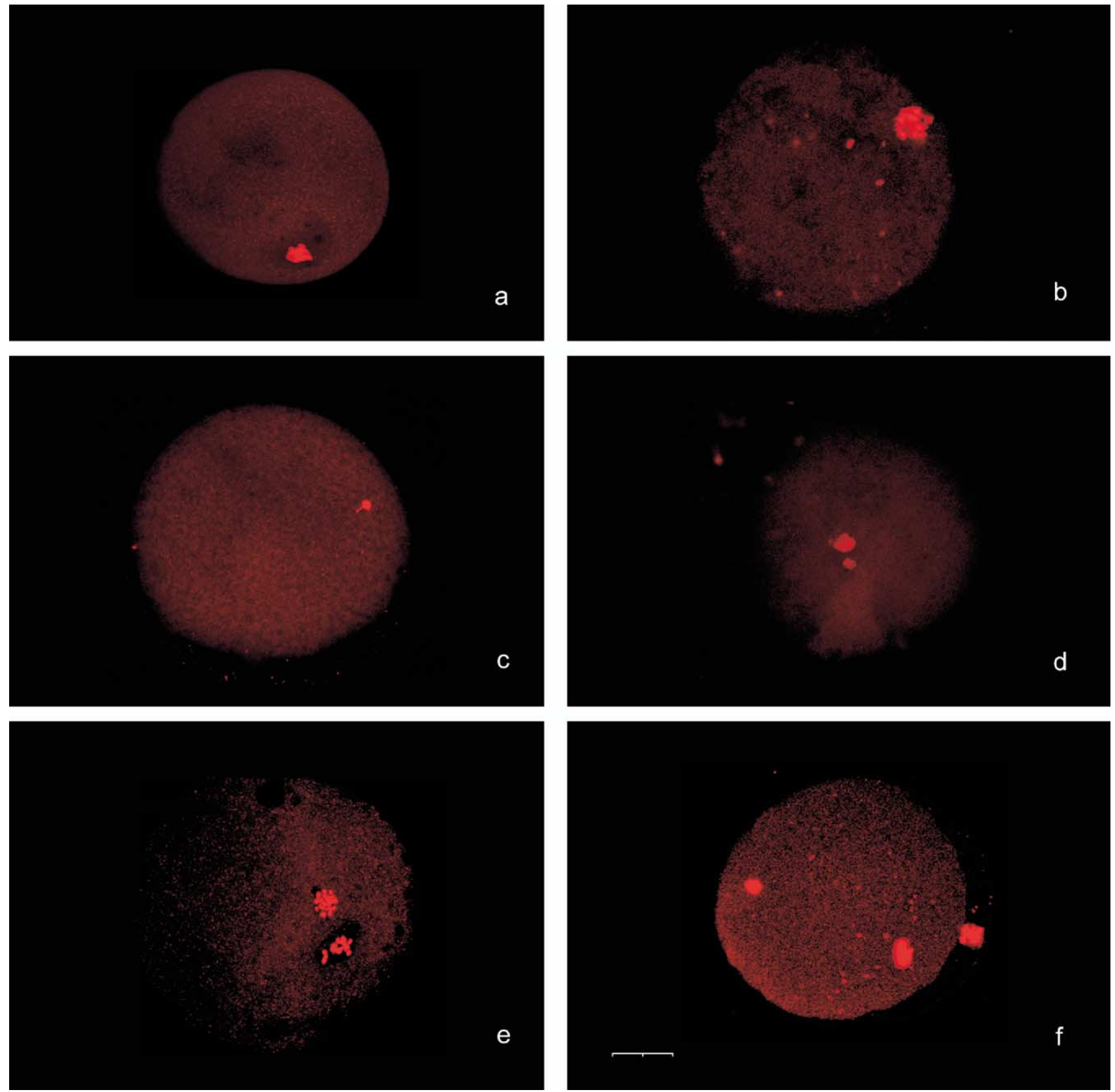

Bosco. Apoptosis in human unfertilized oocytes. Fertil Steril 2005.

essential process for the maintenance of the homeostasis of the ovarian function, both in humans and in other mammal species. In fact, it guarantees the selection of a dominant follicle and the elimination of excessive follicles. This process decreases the possibility of a multiple embryonic de- velopment during pregnancy and ensures the development of fewer but healthy embryos.

Analysis of the morphology has demonstrated that more than $99 \%$ of the ovarian follicles are subjected to so-called 


\section{TABLE 1}

Number and percentage of apoptotic oocytes at different stages of maturation.

Total oocytes undergoing TUNEL assay: 165

Virgin oocytes $(n=84)$

\section{Nuclear \\ maturation (n)}

GV $\quad 12$

MI 6

MII $\quad 66$
Apoptotic oocytes

(\%)

$12(100)$

5 (83.3)

$24(37)^{\mathrm{a}}$

Oocytes unfertilized after ICSI $(n=81)$

Nuclear maturation

(n)
Apoptotic oocytes

(\%)

Note: $\mathrm{MI}=$ metaphase I; $\mathrm{MII}=$ metaphase II; GV = germinal vesicle.

a vs. ${ }^{\mathrm{b}} ; P<.05$.

Bosco. Apoptosis in human unfertilized oocytes. Fertil Steril 2005.

follicular atresia, degeneration processes during reproductive life (20). Two million oocytes are present in the human ovary at birth, but only 400,000 remain by the beginning of puberty. Of these 400,000 only 400 are selected for ovulation, and the remaining majority are subjected to atresia (21). It is more likely that a follicle will die than ovulate. Morphologic analyses have demonstrated that atresia is an irreversible process: once a follicle reaches the point of atresia, it cannot turn back to the ovulatory pathway.

The presence of apoptosis in a pool of cumulus cells from immature oocytes compared with cumulus cells from mature oocytes in metaphase II was studied by Host et al. (22). Their study demonstrated a significant increase in the number of apoptotic cumulus cells in the pool of cells derived from immature oocytes (germinal vesicles and MI) compared with cumulus cells derived from mature oocytes (metaphase II). Although, during reproductive life, apoptosis is strictly related to the reduction of the number of follicles, we think that apoptotic events in follicles selected after hormonal stimulation, which could be related to a lack of oocyte fertilization or low embryo quality, might be triggered in the same way $(23,24)$.

We know that a large number of oocytes recovered after pick-up in assisted fertilization cycles (approximately 35\%) do not complete the meiotic divisions. They remain in the germinal vesicle stage or in metaphase I, despite ovulation induction (determined by using a high concentration of exogenous luteinizing hormone).

The results of our study, with virgin oocytes (not treated with assisted fertilization techniques) and unfertilized oocytes after ICSI, show that apoptosis occurs also in human oocytes retrieved after hormonal stimulation. Interesting data are represented by the percentage of MII oocytes (37\%) that show apoptotic events. This percentage is very similar to the rate of oocyte fertilization failure after ICSI. It may be supposed that these oocytes were probably destined to be physiologically eliminated in the ovary through the process of atresia, which various investigators (3) have demonstrated is closely correlated with apoptotic events.

The data shown in Table 1 for the oocytes treated with ICSI and nonfertilized oocytes demonstrated that fertilization failure, in $88.8 \%$ of the cases, was closely associated with the presence of apoptosis in the oocyte chromatin. Apoptosis was also present in the nemaspermic chromatin of six oocytes. This observation is very important because it demonstrates that spermatozoa with fragmented DNA might nevertheless appear vital and might be injected into the oocyte during ICSI. This is in contrast with the idea that any motile spermatozoon with normal morphologic features is really a healthy spermatozoon.

Compared with other cells, the sperm DNA is very resistant to chemical or physical denaturation. The anomalies of the chromatin packaging in human sperm could be consequent to architectural defects of the nuclear structure as, for example, in a missed substitution of the histones with the protamine during spermatogenesis as it has been shown in some patients $(25,26)$. Such anomalies can be associated with a reduction of the chromatin condensation, with successive increase of the DNA instability and greater sensibility to stress. However, the data seem to demonstrate that the oocyte chromatin plays a main role.

Oocyte apoptosis could be associated with the oocyte's inability to activate itself after sperm injection. A possible explanation could be that the fragmented DNA is associated with a general metabolic defect that leads the oocyte toward a programmed death. Apoptotic events found in virgin oocytes indicate that the process is not a consequence of the injection techniques. However, it cannot be excluded that an apoptotic event could be the consequence of the selection operated by the ovarian stimulation, which allows the retrieval of oocytes from follicles that physiologically would be suppressed by the dominant follicle and assigned to atresia.

As discussed by Van Blerkom (24), DNA fragmentation in oocytes (in particular, GV and MI oocytes) could be a 
In situ immunoreaction of unfertilized oocyte after ICSI. (A) Reaction with the primary antibody caspase-3 cleaved. (B) Stained with propidium iodide. (C) Merged images $A$ and $B$. (D) Merged images of negative control (oocyte incubated without primary antibody caspase-3 cleaved) and stained with propidium iodide. Scale bar $=15 \mu \mathrm{m}$.
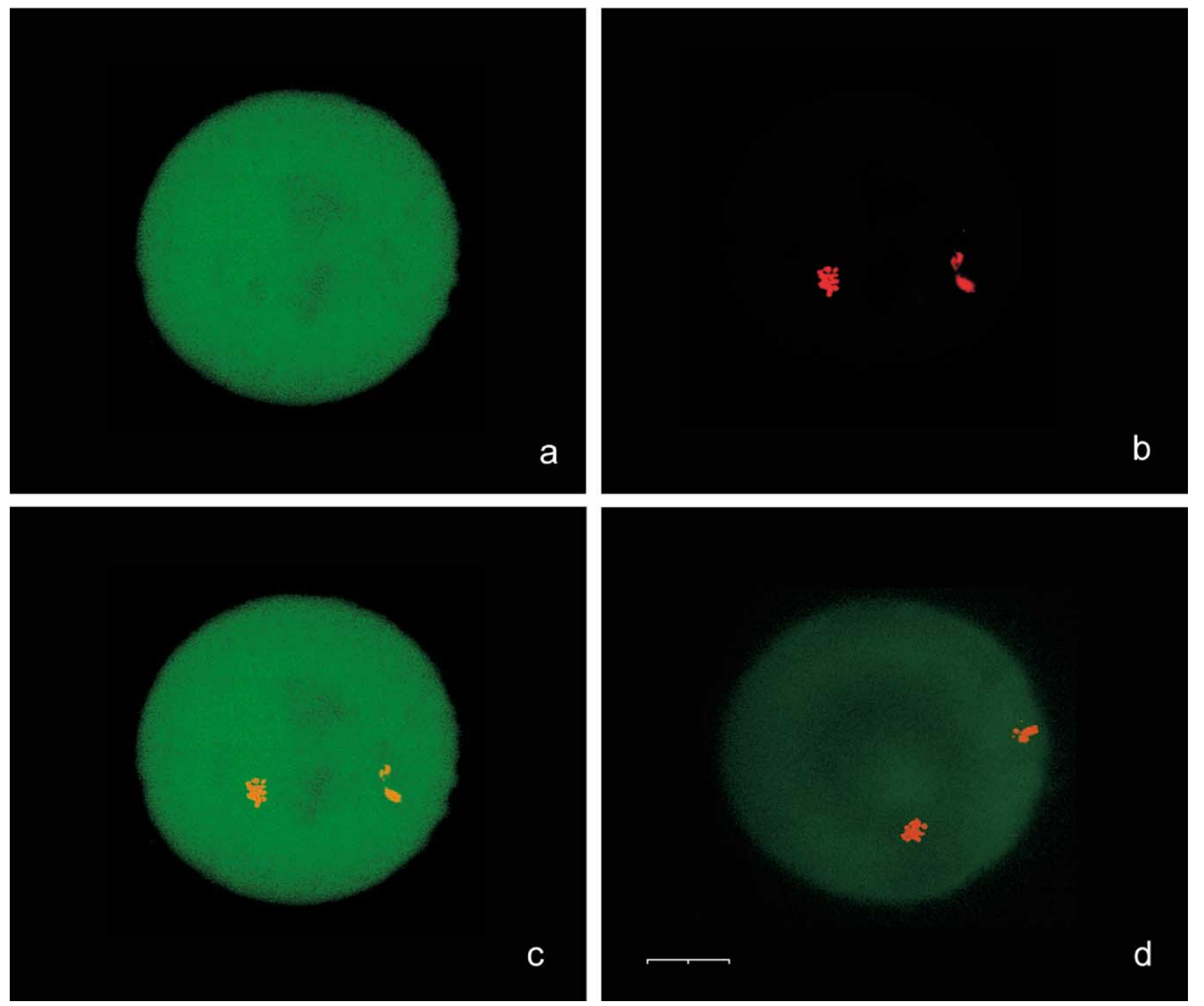

Bosco. Apoptosis in human unfertilized oocytes. Fertil Steril 2005.

consequence of stress during the ovarian follicle maturation or a hypoxic condition resulting from a compromised microcirculation in those follicles correlated to increase aneuploidy rate in the oocytes. So apoptosis could represent a way to eliminate the oocytes with numeral or structural disorders. Apoptosis also could be an expression of the metabolic stress caused by culture conditions, as demonstrated by $\mathrm{Wu}$ et al. (27) in in vitro matured oocytes; however, our study of unfertilized oocytes showing DNA fragmentation and fertilized oocytes that produced embryos used the same culture conditions.
We used the caspase- 3 immunoreaction as a specific test for apoptosis to verify if DNA fragmentation was a consequence of apoptosis pathway. Our study demonstrated that in human oocytes and in some spermatozoa an apoptotic process can be activated. The presence of such events in untreated oocytes-free from stress deriving from in vitro culture or manipulation - could explain the high percentage of fertilization failure after ICSI. Our data demonstrate the presence of DNA fragmentation. To be certain that such evidence is not due to temporary events of DNA repair or transcription, we carried out the analysis for the presence of 
cleaved caspase- 3 , an enzyme certainly involved in the apoptotic process. We think that it is necessary to obtain more data concerning cleaved caspase- 3 and other apoptosis markers, both in nonfertilized human oocytes after ICSI and in oocytes soon after pick-up, to confirm that the presence of apoptosis is an event already in progress and that in some cases it is strictly correlated with fertilization failure.

\section{REFERENCES}

1. Ellis RE, Yuan JY, Horvitz HR. Mechanism and functions of cell death. Ann Rev Cell Biol 1991;7:663-98.

2. Vinatier D, Dufour P, Subtil D. Apoptosis: a programmed cell death involved in ovarian and uterine physiology. Eur J Obstet Gynecol Reprod Biol 1996;67:85-102.

3. Billig H, Assarsson B, Bjornheden D, Svensson E, Thelander H, Utter M. Atresia is an apoptotic process. Frontiers Endocrinol 1995;18:21-34.

4. Oosterhuis GJE, Michgelsen HW, Lambalk CB, Schoemaker J, Vermes I. Apoptotic cell death in human granulosa-lutein cells: a possible indicator of in vitro fertilization outcome. Fertil Steril 1998;70:747-9.

5. Fujino Y, Ozaki K, Yamamasu S, Ito F, Matsuoka I, Hayashi E, et al. DNA fragmentation of oocytes in aged mice. Hum Reprod 1996;11: 1480-3.

6. Schoysman R, Segal L, Van der Zwalmen P, Nijs M, Bertin G, Cittadini E, et al. Fertilization of oocytes by testicular spermatozoa and pregnancy in the human. Acta Eur Fertil 1993;24:103-5.

7. Schoysman R, Segal L, Van Der Zwalmen P, Nijs M, Bertin G, Cittadini E, et al. Assisted fertilization with epididymal spermatozoa. Acta Eur Fertil 1993;24:7-12.

8. Palermo GP, Cohen J, Alikani M. Development and implementation of intracytoplasmic sperm injection (ICSI). Reprod Fertil Dev 1995;7:211-8.

9. Payne D, Matthews CD. Intracytoplasmic sperm injection-clinical results from the reproductive medicine unit, Adelaide. Reprod Fertil Dev 1995;7:219-27.

10. Svalander P, Forsberg AS, Jakobsson AH, Wikland M. Factor of importance for the establishment of a successful program of intracytoplasmic sperm injection treatment for male infertility. Fertil Steril 1995;63:828-37.

11. Evenson D, Darzynkiewica Z, Melamed M. Relation of mammalian sperm chromatin heterogeneity to fertility. Science 1980;240:1131-3.

12. Foresta C, Zorzi M, Rossato M, Varotto A. Sperm nuclear instability and staining with aniline blue: abnormal persistence of histones in spermatozoa in infertile men. Int J Androl 1992;15:330-7.

13. Sailer B, Jost L, Evenson D. Mammalian sperm DNA susceptibility to in situ denaturation associated with the presence of DNA strand breaks as measured by the terminal deoxynucleotidyl transferase assay. $\mathbf{J}$ Androl 1995;15:80-7.

14. Hassold T, Chen N, Funkhouser J. A cytogenetic study of 1000 spontaneous abortions. Ann Hum Genet 1980;44:151-8.

15. Plachot M, Veiga A, Montagut J. Are clinical and biological parameters correlated with chromosomal disorders in early life: a multicenter study. Hum Reprod 1988;627-35.

16. Zenzes MT, Wang P, Casper RF. Cigarette smoking may affect meiotic maturation of human oocytes. Hum Reprod 1995;10:3213-17.

17. Gorczyca K, Gong Y, Darzynkiewicz J. Detection of DNA strand breaks in individual apoptotic cells by the in situ terminal deoxynucleotidil transferase and nick translation assays. Cancer Res 1993;53:1945-51.

18. Roccheri MC, Barbata G, Cardinale F, Tipa C, Bosco L, Oliva O, et al. Apoptosis in sea urchin embryos. Biochem Biophys Res Commun 1997;240:359-66.

19. Roccheri MC, Tipa C, Bonaventura R, Matranga V. Physiological and induced apoptosis in sea urchin larvae undergoing metamorphosis. Int J Dev Biol 2002;46:801-6.

20. Baker TG. A quantitative and cytological study of germ cells in the human ovaries. Proc R Soc Lond B Biol Sci 1963;158:417-33.

21. Byskov AG. Follicular atresia. In: Jones RE, ed. The vertebrate ovary. New York: Plenum, 1978:533-62.

22. Host E, Mikkelsen AL, Lindenberg S, Smidt-Jensen S. Apoptosis in human cumulus cells in relation to maturation stage and cleavage of the corresponding oocyte. Acta Obstet Gynecol Scand 2000;79:936-40.

23. Host E, Gabrielsen A, Lindenberg S, Smidt-Jensen S. Apoptosis in human cumulus cells in relation to zona pellucida thickness variation, maturation stage, and cleavage of the corresponding oocyte after intracytoplasmic sperm injection. Fertil Steril 2002;77:511-15.

24. Van Blerkom J. The influence of intrinsic and extrinsic factors on the developmental potential and chromosomal normality of the human oocyte. J Soc Gynecol Invest 1996;3:3-10.

25. Balhorn R, Reed S, Tanphaichitr N. Aberrant protamine 1/protamine 2 ratios in sperm of infertile human males. Experientia 1988;44:52-5.

26. Manicardi G, Bianchi P, Pantano S. Under protamination and nicking of DNA in ejaculated human spermatozoa are highly related phenomena. Biol Reprod 1995;52:864-67.

27. Wu J, Zhang L, Wang X. Maturation and apoptosis of human oocytes in vitro are age-related. Fertil Steril 2000;74:1137-41. 